\title{
Joint Angle Estimation in Rehabilitation with Inertial Sensors and its Integration with Kinect
}

\author{
Antônio Padilha Lanari Bó, Mitsuhiro Hayashibe, Philippe Poignet
}

\begin{abstract}
In this paper, we explore the combined use of inertial sensors and the Kinect for applications on rehabilitation robotics and assistive devices. In view of the deficiencies of each individual system, a new method based on Kalman filtering was developed in order to perform online calibration of sensor errors automatically whenever measurements from Kinect are available. The method was evaluated on experiments involving healthy subjects performing multiple DOF tasks.
\end{abstract}

\section{INTRODUCTION}

Different applications on rehabilitation and assistive technologies require quantitative assessment of the performed task, such as the measurement of joint motion. Traditionally, depending on the specifications of each particular situation, distinct sensing systems are applied.

Within the clinical or research settings, for instance, optical systems, which normally provide precise estimates, are often used for that purpose. However, these are expensive systems that can only be used to assess the activities performed inside the laboratory environment. Furthermore, they often require the placement of markers on the subject. Recently, however, a inexpensive markerless new system which enables measurements of joint motion without markers has been released, the Kinect system.

On the other hand, for long-term recording of human motion outside clinical facilities, portable sensors that do not rely on external references are an interesting alternative [1]. Among those devices, some of the most popular are inertial sensors, such as accelerometers and gyrometers [2], particularly if assembled as wireless units. Those systems may provide high bandwidth information, but the provided estimates may be corrupted by different types of errors [3].

In order to minimize the effect of those errors, some approaches rely on precise placement of those sensors on the body segments [4], which may be a limiting factor for some applications. Another approach then is to rely on the complimentary features of both system and compute the best estimate using sensor fusion. Indeed, other portable sensors, such as magnetometers, may be included in the integration in order to improve the overall quality [5].

During this work, Antônio P. L. Bó (e-mail: antonio.plb@gmail.com), Mitsuhiro Hayashibe and Philippe Poignet were with the LIRMM, France. Antônio P. L. Bó is now at the Universidade the Brasília, Brazil. Mitsuhiro Hayashibe is also with the INRIA, France.
Based on this scenario, the motivation of the current paper is the exploration of the combined use of inertial systems and the Kinect to estimate human motion. This integration has the potential of minimizing the drawbacks of both individual systems by enabling faster system initialization, better visualization of the estimated motion, increasing the bandwidth and improving the overall precision.

In this work, we have used simple motion sensing units composed of a 3 -axis accelerometer and a 2-axis gyrometer to estimate joint angles in different tasks that illustrate common rehabilitation applications. Data from both sensors are integrated using a Kalman Filter (KF), for which we propose a new method for taking into account errors in the accelerometers estimates. Furthermore, a method based on the same KF framework is proposed to use the joint angles provided by the Kinect to correct temporarily the overall estimate and calibrate the inertial sensors to enable long-term operation without the optical system. The complete method was then evaluated on tests conducted on healthy subjects.

\section{MethoD}

\section{A. Sensors}

1) Inertial sensors: Two types of inertial sensors were used in this work, accelerometers and gyrometers. Both devices present the clear advantage of reduced size, weight and cost, but often at the price of providing measurements corrupted with different types of sensing inaccuracy, such as sensitivity errors and time-varying bias.

The term accelerometer usually refers to sensors whose output depend on both the inertial acceleration and the gravitational acceleration onto the device sensitive axis. Here we are mainly interested in using the gravity component measured by accelerometers to estimate the joint angle. For that reason, we wish to neglect the measured inertial acceleration, which may be seen as a disturbance in this case.

Concerning the gyrometers, these devices measure the angular rate with respect to an inertial frame. Hence, the main problem when using gyrometers to compute rotational displacement is that small errors on the sensor reading propagate in time, since the estimates are obtained using numeric integration.

2) Kinect: Microsoft's Kinect is a low-cost device for human-computer interface composed by a RGB camera and a depth sensor based on an infrared camera. Using 


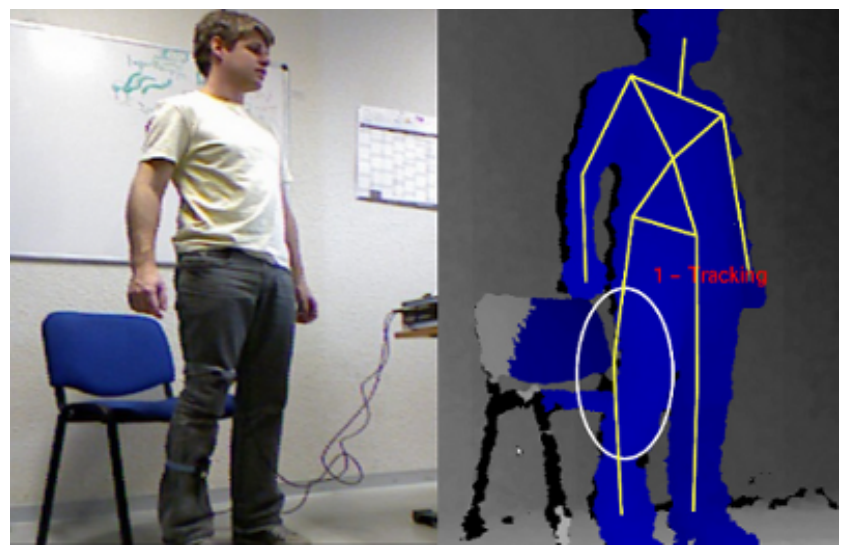

Fig. 1. Estimation of 3D joint positions using Kinect. The white ellipse indicated a typical error originated from the depth-based skeleton reconstruction. Also, it may be observed that the chair is recognized as the part of left leg.

appropriate software, such as the Primesensor NITE Middleware, the system computes useful high-level measurements related to the user and her motion.

In this work, the device is considered as a sensor which provides directly the preprocessed data required for integration with other sensors. More specifically, two types of information are retrieved from the system. First, 3D joint positions of the user (illustrated in Fig. 1) are used to compute the angles of the joints of interest. For instance, in order to compute the relative angle $\theta$ between two vectors measured by Kinect, such as $\mathbf{p}_{1}$ and $\mathbf{p}_{2}$, we may simply do

$$
\theta=\arccos \left(\frac{\mathbf{p}_{1} \cdot \mathbf{p}_{2}}{\left\|\mathbf{p}_{1}\right\|\left\|\mathbf{p}_{2}\right\|}\right) .
$$

Secondly, the information from Kinect is used to provide $3 \mathrm{D}$ visualization of the results obtained with the sensing systems. This feature indeed is particularly useful for studying human motion for rehabilitation purposes, since it gives an intuitive interface to validate if motion estimation with inertial sensors is correctly performed in the physical space from different points of view. The $3 \mathrm{D}$ joint position reconstruction with Kinect is based on the module developed by Primesense. We have developed the environment to integrate the spaces of inertial sensors and Kinect/normal video camera with the camera calibration using OpenCV.

The technology which the device is based on was originally developed by Primesense, which also developed the software used in this work. However, since the system has been more commonly referred to as Kinect, this is the designation that has been adopted in this work.

\section{B. Motion sensing and rehabilitation tasks}

The different tasks that are often conducted during physical therapy, research on posture and gait, and other related situations impose distinct requirements for the sensing systems used for measuring joint motion. For instancem one of the most important requirements for such systems concerns the subject's safety. Indeed, in the case of close interaction between rehabilitation or assistive devices with the subject, such a system must guarantee a high level of confidence of those estimates. For instance, if gyrometers are used to estimate joint motion, this normally indicates then that the operation time of the device will be rather limited, due to the drift in time of the estimates provided by such systems.

Furthermore, those systems must also be easy to setup, since long initialization or calibration procedures may lead to less successful therapies when compared to "plugand-play"' devices. With respect to that specification, portable sensors are less practical comparing to the Kinect, for instance. Indeed, not only those portable systems must be placed on the corresponding body parts, but also a re-calibration procedure must be performed, since changes on the attachment of the devices on the segments are inevitable.

On the other hand, considering the use of those systems for different daily tasks, the advantages of using inertial systems for measuring human motion are evident, since valuable information may be retrieved using data acquired during real life activities on long-term recordings outside the laboratory environment. Systems such as the Kinect also presents irregular performance on nonstructured environments, as illustrated in Fig. 1.

Based on that discussion on sensing systems for rehabilitation tasks, the benefits of the combined use of inertial sensors and the Kinect become more clear. Inertial sensors are indeed quite useful for rehabilitation purposes, but the difficulties concerning initialization and the constant need to re-calibrate the readings prevent a broader use. In this scenario, Kinect may be a valuable calibration tool which stays at home and/or in the clinical setting. In order to evaluate that integration we have chosen the following tasks:

- Sit to stand (knee, 1 DOF, and ankle, 1 DOF)

- Squat (knee, 1 DOF, and ankle, 1 DOF)

- Shoulder abduction/adduction (1 DOF)

\section{Integrating inertial sensors measurements}

The integration of inertial sensors and the Kinect in this work is described in subsequent steps, and we start by presenting the fusion of accelerometers and gyrometers. Indeed, for joint motion estimation, these two sensors have complimentary roles. Gyrometers estimates are affected by the accumulated effect of sensor errors, but the sensor readings are seldom affected by other disturbances. On the other hand, the estimates provided by the accelerometers do not present any drift in time, but they are highly sensitive to disturbances, such as inertial accelerations of the device. Moreover, joint motion estimates using accelerometers from rotations around gravity are impossible to obtain.

In this scenario, a well-known method to improve the quality of the overall estimate is to fuse the information obtained from both sensors using stochastic 
filters. In this work, a Kalman filter (KF) has been used for that purpose. As an example, we will describe the integration concerning a 1 DOF task, such as shoulder abduction/adduction.

For this motion, the joint angle estimates obtained using inertial sensors are given by

$$
\begin{aligned}
\theta_{g} & =\int \tilde{\omega}_{y} d t \\
\theta_{a} & =\alpha \arccos \left(\frac{f_{x}}{\|\mathbf{g}\|}\right)+(1-\alpha) \arcsin \left(\frac{f_{z}}{\|\mathbf{g}\|}\right),
\end{aligned}
$$

where $\mathbf{g}$ is the magnitude of the local gravity field, $g$ and $a$ refers respectively to gyrometer and accelerometer, and $\alpha$ is related to the relative confidence on the measurements provided by the $x$ and $z$ axes of the 3 -axis accelerometer.

Within the KF framework, the estimation is organized in prediction and correction phases [6]. In the present case, the initial a priori estimate is computed according to Eq. (2), while the correction is given by the estimate provided by the accelerometer (Eq. (3)). We have chosen to apply a loosely coupled integration scheme, i.e., the data applied to the filter is previously processed, such that minimal changes have to be made to the filter in order to represent different tasks.

Regarding the parameters that may be used to tune the behavior of the algorithm, the most important parameters are the process variance $q$ (or the process covariance matrix $\mathbf{Q}$ for motions with multiple DOF) and the measurement variance $r$ (or, similarly, the measurement covariance matrix R). Process variance, which in this case represents the uncertainties related to the gyrometer, is chosen based on the sensor noise. Likewise, $r$ is chosen based on the accelerometer noise. Concerning the accelerometer, however, since measurements of the gravitational field are affected by inertial accelerations performed by the device, its estimates may contain incorrect information. In order to minimize this effect, instead of using a fixed value of $r$, we have applied a variable variance $r$ which depends on the overall measured acceleration. It is given by

$$
r(\tilde{\mathbf{f}})=r+\lambda\left(\frac{\exp \left(\beta\left\|r_{i}(\tilde{\mathbf{f}})\right\|\right)-1}{\exp (\beta)}\right),
$$

where $\beta$ and $\lambda$ are tuning constants, and

$$
r_{i}(\tilde{\mathbf{f}})=\frac{\|\mathbf{g}\|-\|\tilde{\mathbf{f}}\|}{\|\mathbf{g}\|} .
$$

\section{Online calibration using inertial sensors and Kinect}

Although the integration described in the previous section may highly improve the quality of the overall estimate, significant errors on the motion computed may still occur. Here we propose to include the Kinect as an additional correction within the filter, in order to minimize some of these undesirable effects.

One of those typical problems refers to the definition of the initial conditions of the algorithm, i.e., the initial states and covariances related to each sensor or device. This problem is directly and easily solved using Kinect. In the experiments described in this work, for instance, the estimates provided by the Kinect were used to initialize the integration filter.

Another problem that demands further attention, however, refers to the errors in the sensors readings, an issue that is partially solved by the filter described in Sec. II-C. However, if sensor errors increase, unsatisfactory results will be inevitably produced. Furthermore, often those devices need to be constantly calibrated, particularly if low-cost devices are used. For those reasons, in this work we propose to use the Kinect to perform online calibration of inertial sensors, whenever its measurements are available.

In order to perform this online calibration, we consider that the main source of error on angle estimation using inertial sensors is the bias $b$, which is assumed to be a random walk process. Then, estimation of the gyrometer bias may be performed by including the corresponding bias within the state vector, thus extending the filter described in the previous section. This augmentation will result on the following extended model for joint estimation using a gyrometer:

$$
\theta(k)=\theta(k-1)+\left(\tilde{\omega}(k-1)-b_{\omega}(k-1)\right) T_{s},
$$

where $T_{s}$ is the sampling period.

This simple extension to the filter is capable of compensating typical undesirable phenomena related to gyrometers, such as time-varying bias caused by small sensor displacements. Hence, if both the measurements of the accelerometer and the Kinect become unavailable during the trial, the estimates from the gyrometer solely will provide a better performance when compared to the uncalibrated device.

Concerning the accelerometer, a different approach with respect to the gyrometer is adopted in order to simplify the design. Since the model for joint estimation using a accelerometer (Eq. (3)) is considerably more complex than its analogous using a gyrometer (Eq. (2)), the strategy is to estimate the offset between the angle estimated by the Kinect, $\theta_{k}$, and $\theta_{a}$. This value $e_{\theta}$ is then modeled as a random walk process and its value is then estimated from within the filter, resulting in an extended state vector of size 3 , i.e., $\left[\begin{array}{lll}\theta_{g}(k) & b_{\omega}(k) & e_{\theta}(k)\end{array}\right]^{T}$. The value of $e_{\theta}$ is then used to correct the angle estimate from the accelerometer (Eq. (3)), considerably improving the system performance once the Kinect measurements become unavailable.

\section{EXPERIMENTS}

\section{A. Setup}

Series of experiments were designed to evaluate the methods discussed in this work ${ }^{1}$. The trials involved

\footnotetext{
${ }^{1}$ A video from the experiments illustrating the method and the $3 \mathrm{D}$ visualization using Kinect is available online at http://www . lirmm.fr/ hayashibe/IMU/embc2011.wmv.
} 


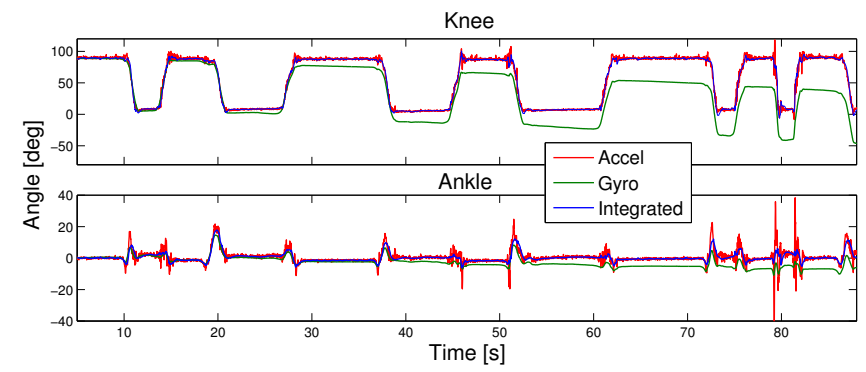

Fig. 3. Sit-to-stand estimation using gyrometers and accelerometers only.

healthy subjects performing tasks described in Sec. IIB. The subjects were allowed to move freely within the task limits. In Fig. 2 a subject performing the sit-to-stand task is illustrated.

The hardware used in the experiments is composed by:

- Inertial motion sensing units composed by 2-axis gyrometers, such as the IDG-300 and the IDG500 , angular rate sensors from Invensense, and the MMA7260QT, a 3-axis accelerometers from Freescale. Measurements from both sensors are acquired using a National Instruments acquisition card.

- Microsoft's Kinect system for human-computer interface using the Primesensor NITE Middleware provided by Primesense.

\section{B. Results}

The first tests conducted in this work were performed to validate the basic algorithm presented in Sec. II$\mathrm{C}$ for multiple DOF movements. Figure 3 illustrates a sit-to-stand experiment, which clearly exemplifies the individual errors from both gyrometers (accumulated error due to bias) and accelerometers (noise and inertial acceleration peaks) and also shows the improvements in performance obtained fusing both estimates.

In the experiment illustrated in Fig. 3, it was considered that the subjects started the task in a resting position, from where changes in angle were computed. In Fig. 4, which refers to a squat movement, the the system initialization was performed using the Kinect. Also, the angles estimated with the Kinect may also be used to validate the estimates obtained using the inertial sensors, which illustrates the coherence of the angles computed from the inertial sensors.

Finally, in Fig. 5 we illustrate the performance obtained when both the Kinect and the inertial sensors are used, and online calibration of the sensors is performed. In order to enable a better visualization of the benefits of the method, online calibration is performed only in the first half of the data. By doing so, we emulate the situation where the measurements from the Kinect are no longer available. On the top figure, the improvements on the estimates from the gyrometer are shown, while

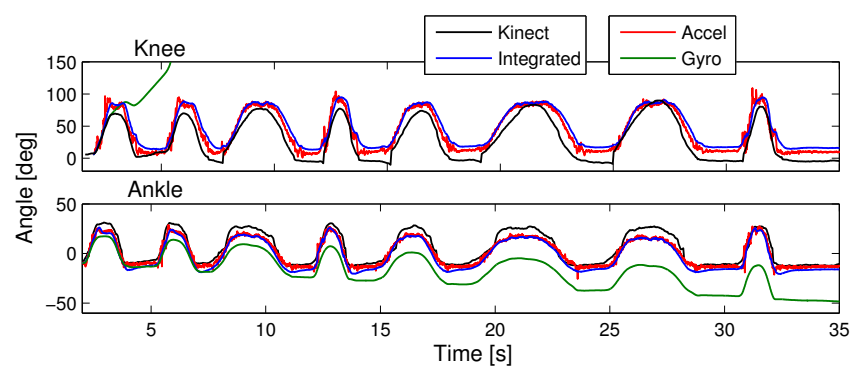

Fig. 4. Squat motion estimation using gyrometers and accelerometers only compared with the estimates provided by the Kinect. The consistency of the integrated estimate may be observed, as well as the effect of large bias error in the gyrometer (knee angle).

the bottom figure illustrates the differences between the uncalibrated accelerometer and the one calibrated online.

\section{Discussion}

The results shown in Figs. 3 and 4 indicate that fusing the estimates of gyrometers and accelerometers may provide sufficient high performance without further need of precise calibration. However, in order to obtain such satisfactory response when larger sensor errors are present, suitable calibration is required. The minimization of such errors may be difficult, since low-cost sensors often present time-varying parameters and also because the sensing units frequently displace from their initial position on the body. Additionally, in some cases joints of interest may be not observable using accelerometers, a case in which solely the estimation from gyrometers would be considered.

For both types of errors, the use of external systems such as the Kinect may be extremely helpful for conducting brief online calibration periods, as illustrated in Fig. 5. Indeed, interesting feature of the method enables the following experimental flow for experiments requiring joint motion estimation, which may be of great interest for applications on rehabilitation:

1 install portable sensors

2 perform preliminary movements in front of Kinect, if an initial calibration is required

3 while sensing system is on do

$4 \quad$ if Kinect is present then

$5 \quad$ online calibration is performed

6 else

$7 \quad \mid$ estimate from inertial sensors only

8 end

9 end

On the other hand, there are still some issues concerning the use of the Kinect in the form it was used in our trials. Firstly, clear errors on the joint positions provided by the Kinect were observed on several occasions, as illustrated in Fig. 1. These errors occurred even in situations where no occlusions were present or complex 

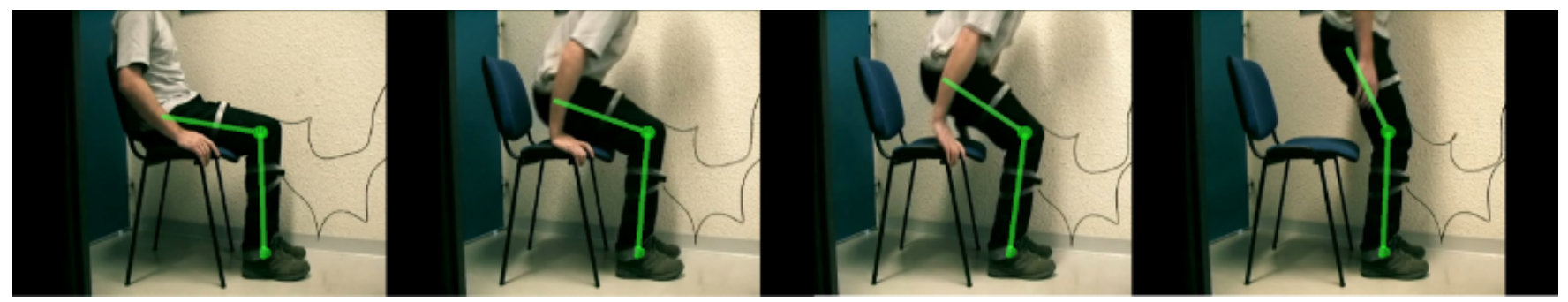

Fig. 2. Angles estimated using inertial sensors in a sit-to-stand task.

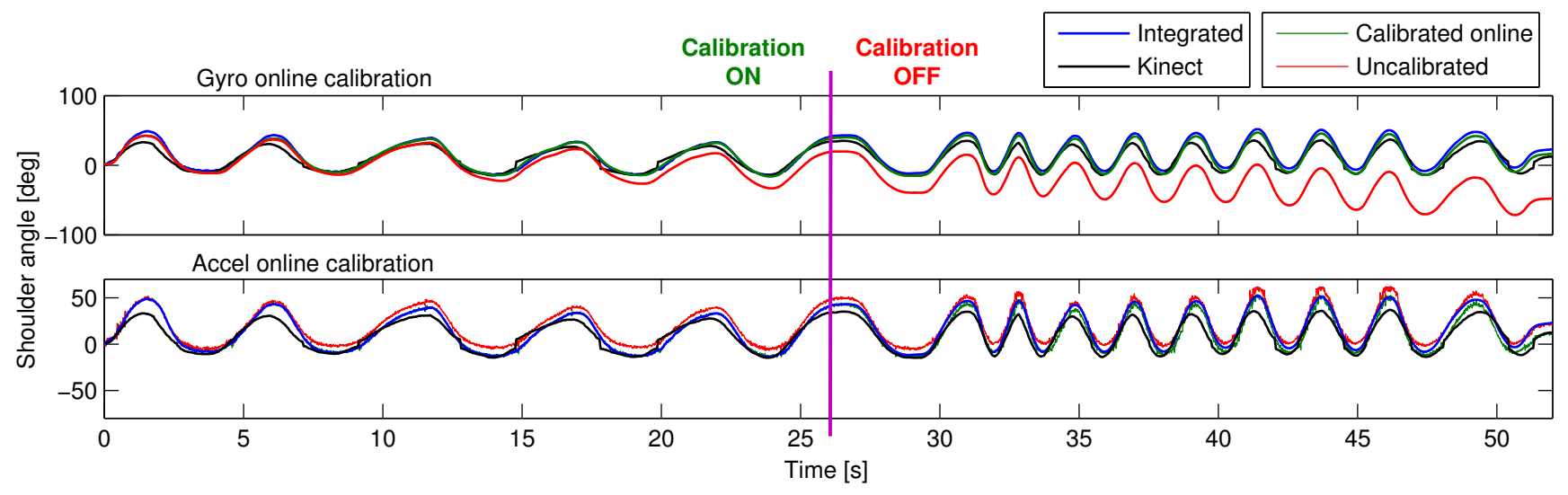

Fig. 5. Shoulder abduction/adduction estimation using gyrometers, accelerometers and the Kinect. In the first half of the trial, the Kinect was used to calibrate online the inertial sensors. For the second half, the values obtained in this initial calibration were kept constant. In both figures, the Kinect data (black) and the integrated estimate (blue) are the same. The remaining data (green and red) represent the effect of online calibration on the gyrometer (top) and the accelerometer (bottom).

movements were performed. Secondly, the periods between the provided measurements were seldom constant and difficult to control, which complicates the design of integration algorithms. These issues are related to the combination of driver and middleware used in this work. Future software versions may reduce those effects on the system performance.

\section{Conclusion AND Future WORKS}

The potential of the combined use of portable sensors (such as accelerometers, gyrometers and magnetometers) and the Kinect for measuring human motion for rehabilitation purposes is tremendous. The Kinect enables simpler initialization procedures, a better visualization of the estimated angles, and the possibility of calibrating the inertial sensors in real-time. On the other hand, the Kinect also presents limitations, such as the limited workspace, estimation errors due to occlusions and other situations (e.g., Fig. 1), and the current sampling frequency obtained from the device, which is inadequate for faster movements.

In this scenario, in this work firstly the Kinect was employed as a reference for the inertial system initialization and for improving the visualization of motion in 3D. Afterwards, we explored the problems related to measurement errors from inertial sensors and proposed a new method based on Kalman filtering to integrate the available information in order to improve the overall estimated motion. The method was evaluated on experiments involving healthy subjects and satisfactory results were obtained.

Further work will focus on improving extend the online calibration filter to include further sensor errors, such as scaling, and evaluating integration of Kinect and portable sensors for motion capture system with more DOF. Moreover, the system will be applied in long-term clinical trials to evaluate acceptance and performance.

\section{REFERENCES}

[1] C. V. Bouten, K. T. Koekkoek, M. Verduin, R. Kodde, and J. D. Janssen, "A triaxial accelerometer and portable data processing unit for the assessment of daily physical activity." IEEE Transactions on Biomedical Engineering, vol. 44, no. 3, pp. 136-147, mar. 1997.

[2] D. H. Titterton and J. L. Weston, Strapdown inertial navigation technology. Peter Peregrinus LTD., 1997.

[3] P. Cheng and B. Oelmann, "Joint-angle measurement using accelerometers and gyroscopes - a survey," IEEE Transactions on Instrumentation and Measurement, vol. 59, no. 2, pp. 404 -414 , feb. 2010.

[4] H. Dejnabadi, B. Jolles, and K. Aminian, "A new approach to accurate measurement of uniaxial joint angles based on a combination of accelerometers and gyroscopes," IEEE Transactions on Biomedical Engineering, vol. 52, no. 8, pp. 1478 -1484, aug. 2005 .

[5] A. P. L. Bó and G. A. Borges, "Low cost 3d localization system for application on aerial robots," in Symposium Series in Mechatronics, P. E. Miyagi, O. Horikawa, and J. M. Motta, Eds. Associação Brasileira de Engenharia e Ciências Mecânicas, 2008, vol. 3, pp. 355-362.

[6] D. Simon, Optimal State Estimation: Kalman, H Infinity and Nonlinear Approaches. John Wiley \& Sons, Inc., 2006. 